\title{
25 Research Suare \\ Comparison of OPD-Referral and Self-Referral Patients In The Emergency Department
}

\author{
Yu-Rung Chou \\ National Cheng Kung University Hospital \\ Mi-Chia Ma \\ National Cheng Kung University \\ Ching-Chi Lee \\ National Cheng Kung University Hospital \\ Chih-Chia Hsieh \\ National Cheng Kung University Hospital \\ Chih-Hao Lin ( $\nabla$ emergency.lin@gmail.com ) \\ National Cheng Kung University Hospital
}

\section{Research Article}

Keywords: acute care, clinical assessment, emergency department, emergency department management

Posted Date: November 19th, 2021

DOI: https://doi.org/10.21203/rs.3.rs-1069600/v1

License: (c) (i) This work is licensed under a Creative Commons Attribution 4.0 International License. Read Full License 


\section{Abstract}

Background: To compare outpatient department (OPD) referral patients and self-referral patients in the emergency department (ED) in terms of hospitalization, mortality, and length of stay.

Methods: We collected ED patients of a tertiary teaching hospital over a 3-month period. We excluded pediatric patients and patients with certain inconsistent characteristics, such as trauma and out-ofhospital cardiac arrest, or referral from other facilities. After propensity score matching, we compared the hospitalization, mortality, and length of stay in the ED of the OPD-referral patents and self-referral patients. We categorized the patients as "emergency" or "urgency" according to their triage information and then analyzed the effects of different severity levels.

Results: The OPD-referral ED patients, compared with the self-referral patients, had a higher admission rate $(49.8 \%$ vs. $28.9 \%, p<0.001$; odds ratio [OR] = 2.44, 95\% confidence intervals [Cls]: 1.91 - 3.12). Among the emergency patients, there was no significant difference regarding the admission rate $(62.6 \%$ vs. $55.8 \%, p=0.257)$ or the mortality rate $(4.6 \%$ vs. $8 \%, p=0.253)$. Among the urgent patients, the admission rate was significantly different between the OPD-referral and self-referral groups ( $46 \%$ vs. $20.2 \%, p<0.001 ; O R=3.36,95 \%$ Cls: $2.48-4.55)$. The urgent patients who were referred from OPD tended to have a higher mortality rate $(2.1 \% \mathrm{vs} .0 .5 \%, p=0.064)$. Regarding the length of ED stay, only the discharge and urgent subgroups differed according to OPD- and self-referral status $(p<0.001)$, with a median of 5.8 hours versus 2.3 hours.

Conclusions: The OPD-referral ED patients have a higher admission rate and a longer length of stay than self-referral patients in urgent triage. The OPD-referral ED patients might have more severe and complex conditions. We should be more alert to OPD-referred patients even when they initially appear not severely ill.

\section{Background}

For many patients, emergency departments (EDs) provide important access to medical care resources. Patients may approach the EDs in several ways, including by self-referral, ambulance transport, and transfer from the outpatient department (OPD) or other medical institutes. Self-referral patients come to EDs based on their own judgment. They believe that their illness is so critical that it is necessary to go to the EDs, rather than general physicians, for medical help. Only half of self-referral ED patients were categorized as appropriate [1]. The inappropriateness of ED visits may include patients who desire to be cured quickly, who ask for doctors' opinions, who are already on waiting list of scheduled surgery, who don't trust their primary care physician, or who is asking for diagnostic tests to rule out major diseases [1-3]. These could induce the ED overcrowding and even delay the urgent managements of critical patients $[1,2,4,5]$.

Patients who are transferred from the OPD are evaluated by other doctors before their arrival at the ED. They are transferred to EDs on a doctor's recommendation. In a survey among Australian general 
practitioners, $0.5 \%$ of individual problems that the general practitioners handled resulted in ED referral [6]. The clinical challenges that require ED referral emphasize the importance of continuity of care for acute presentations. A study in Norway concluded medical necessity in $93 \%$ of the referrals from general practitioners [7]. However, significant differences were observed in reasons for referral among general practitioners, which might reflect difficulties in dealing with professional uncertainty (7).

The major difference between these self-referral patients and OPD-referral patients is whether they were evaluated by a doctor prior to their arrival at the ED. Sometimes, patients already underwent imaging and laboratory studies at the OPD, which can be read by electronic medical record system and facilitate the progression in the ED. The reasons for transferring include unstable physiological conditions, the need for supportive care or specific treatment, and further observation of illnesses.

The OPD-referrals may have specific purposes and implications. Compared with self-referred patients in the ED, the OPD-referral patients might have different presentations. We hypothesized that patients who were transferred from the OPD were more critical and required more medical care resources than selfreferral patients. In this study, we aimed to investigate these two groups of patients. To understand the characteristics of the OPD-referral patients may improve the care of these patients.

\section{Methods}

This was a retrospective study of all nontraumatic adult patients ( $\geq 18$ years of age) who visited the ED of a tertiary teaching hospital between August 1, 2020 and October 31, 2020. There are roughly 100,000 ED visits annually. Patient data were extracted from the electronic medical records system.

Study design and subjects

During the study period, nontraumatic adult patients who visited the ED were enrolled. Patients with outof-hospital cardiac arrest were excluded due to their extremely critical conditions. Patients who left the hospital against medical advice or were transferred to another hospital were also excluded because it was difficult to trace these patients' outcomes. Patients who were referred from other medical institutions were also excluded.

Based on how the patient was referred to the ED, the patients were divided into two groups. Patients who came to the ED directly were designated the self-referral group, while patients who were referred by a doctor from the OPD were designated the OPD-referral group. In each group, the patients were divided into two subgroups in accordance with the triage results. Triage was based on the Taiwan Triage and Acuity Scale (TTAS), which is a 5-level triage system from level 1, the most critical illness, to level 5, nonurgent. The patients triaged as level 1 and level 2 were designated the emergency group. The other patients were designated the urgent group (Table S1).

We performed propensity score matching to diminish the effects of confounding variables on outcomes. The propensity score was calculated using factors related to the patient's age, sex, acute illness, and 
basic health condition. The triage level and the Quick Sequential Organ Failure Assessment (qSOFA) score were used to describe acute illness. The patient's basic health condition was evaluated based on his or her personal history of several common and significant diseases, including coronary artery disease, congestive heart failure, diabetes, hypertension, end-stage renal disease, and malignancy.

\section{Outcomes}

The primary outcome was hospitalization and mortality. Hospitalization was defined as either admission to the hospital or in-ED death. Mortality was defined as in-hospital death within 30 days, including in-ED death. The secondary outcome was the length of stay (LoS) in the emergency department, defined as the interval between ED arrival and leaving the ED due to either admission or going home.

\section{Statistical analysis}

The data were analyzed with the Statistical Package for the Social Sciences for Windows, Version 23.0 and $\mathrm{R}$ language. Categorical variables were changed to numbers with individual definitions. The data were compared by the Pearson's chi-square test or the fisher exact test when applicable, statistically significant were considered when the $p<0.05$. Because the length of stay data did not fit the normal distribution, we used medians and interquartile ranges (IQRs) to describe the distribution of the length of stay. These data were analyzed with the Kruskal-Willis test, and a $p<0.05$ was considered significant.

\section{Results}

During the study period, 20,636 patients visited the ED. We excluded 2,214 pediatric patients ( $<18$ years old), 254 patients discharged against medical advice, 27 who left the hospital without a formal discharge process, 363 patients who were transferred to another hospital for admission or operation and 65 out-ofhospital cardiac arrest patients. A total of 3,751 traumatic cases were excluded because of considerable deviation arising from the fact that the OPD-referred trauma patients usually had chronic problems that were already under a physician's care, while self-referred trauma cases were likely to be new injuries and emergent situations. A total of 1,371 cases were referred from local medical clinics (LMDs) or other hospitals and thus were excluded. Although these patients had conditions similar to those of OPDreferred patients, they came from different hospital systems and sometimes arrived at our hospital with different image files and indications that could affect our management and the patient's length of stay. Sixty-eight visits were excluded because they did not have vital signs recorded at triage which caused the incalculable of qSOFA scores. The patient flow diagram in this study was depicted in Figure 1.

Of the remaining 12,523 eligible cases, 564 patients were referred from our own OPD and comprised the OPD referral group, while the remaining 11,959 patients comprised the self-referral group (Table 1). The self-referral group was younger ( $53.9 \pm 21.2$ vs. $60.6 \pm 18.2$ years), and a higher percentage was triaged as less urgent $(2.78 \%$ vs. $0.18 \%)$ and nonurgent ( $0.46 \%$ vs $0 \%)$. No significant different was noted in qSOFA scores. All comorbidities were more common in the OPD-referral group. 
Table 1

Summary of patient characteristics.

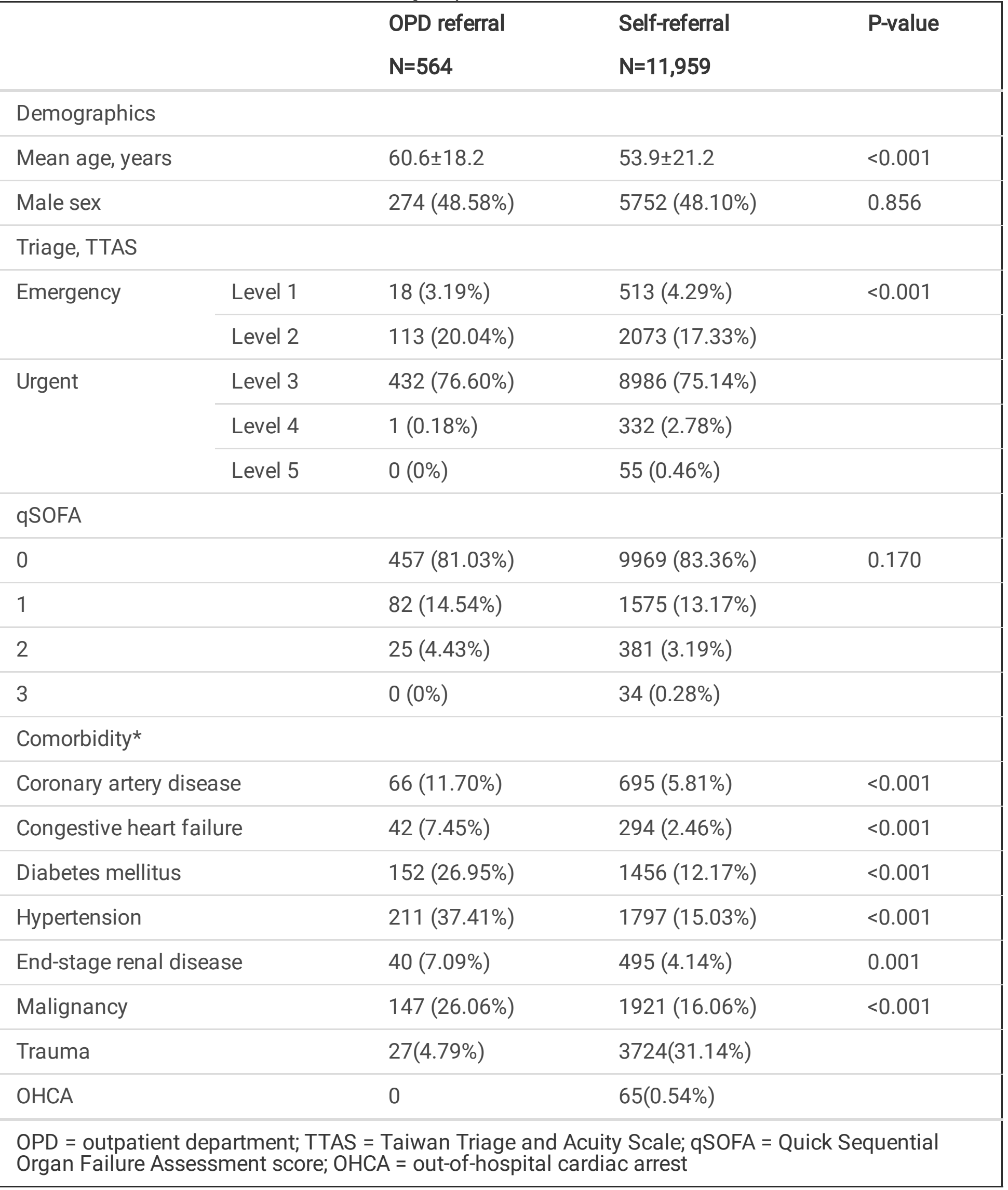


To better compare these two groups, we matched 564 self-referral and OPD-referral cases. With logistic regression and nearest-neighbor matching, we identified 564 self-referral patients with characteristics similar to those of the OPD-referral group (Table 2).

Table 2

Patient characteristics after 1:1 propensity score matching.

\begin{tabular}{|c|c|c|c|c|}
\hline \multicolumn{2}{|l|}{ 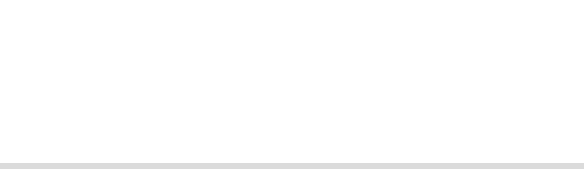 } & $\begin{array}{l}\text { OPD referral } \\
N=564\end{array}$ & $\begin{array}{l}\text { Self-referral } \\
N=564\end{array}$ & P-value \\
\hline \multicolumn{5}{|l|}{ Demographics } \\
\hline \multicolumn{2}{|l|}{ Mean age, years } & $60.6 \pm 18.2$ & $60.4 \pm 18.2$ & 0.861 \\
\hline \multicolumn{2}{|l|}{ Male sex } & $274(48.58 \%)$ & $279(49.47 \%)$ & 0.812 \\
\hline \multicolumn{5}{|l|}{ Triage, TTAS } \\
\hline \multirow[t]{2}{*}{ Emergency } & Level 1 & $18(3.19 \%)$ & $17(3.01 \%)$ & 0.916 \\
\hline & Level 2 & $113(20.04 \%)$ & $121(21.45 \%)$ & \\
\hline \multirow[t]{3}{*}{ Urgent } & Level 3 & $432(76.60 \%)$ & $425(75.35 \%)$ & \\
\hline & Level 4 & $1(0.18 \%)$ & $1(0.18 \%)$ & \\
\hline & Level 5 & $0(0 \%)$ & $0(0 \%)$ & \\
\hline \multicolumn{5}{|l|}{ qSOFA } \\
\hline \multicolumn{2}{|l|}{0} & 457 (81.03\%) & $462(81.91 \%)$ & 0.667 \\
\hline \multicolumn{2}{|l|}{1} & $82(14.54 \%)$ & $83(14.72 \%)$ & \\
\hline \multicolumn{2}{|l|}{2} & $25(4.43 \%)$ & $19(3.37 \%)$ & \\
\hline \multicolumn{2}{|l|}{3} & $0(0 \%)$ & $0(0 \%)$ & \\
\hline \multicolumn{5}{|l|}{ Comorbidity } \\
\hline \multicolumn{2}{|l|}{ Coronary artery disease } & $66(11.70 \%)$ & $59(10.46 \%)$ & 0.569 \\
\hline \multicolumn{2}{|l|}{ Congestive heart failure } & $42(7.45 \%)$ & $40(7.09 \%)$ & 0.909 \\
\hline \multicolumn{2}{|l|}{ Diabetes mellitus } & $152(26.95 \%)$ & $157(27.84 \%)$ & 0.789 \\
\hline \multicolumn{2}{|l|}{ Hypertension } & $211(37.41 \%)$ & $218(38.65 \%)$ & 0.713 \\
\hline \multicolumn{2}{|l|}{ End-stage renal disease } & $40(7.09 \%)$ & $44(7.80 \%)$ & 0.734 \\
\hline \multicolumn{2}{|l|}{ Malignancy } & $147(26.06 \%)$ & $139(24.65 \%)$ & 0.632 \\
\hline
\end{tabular}


The admission and mortality rates of the OPD-referral group and self-referral group were $49.8 \%$ vs. $28.9 \%$ $(p<0.001$, odds ratio [OR] $=2.44,95 \%$ confidence interval [Cls]: $1.91-3.12)$ and $2.7 \%$ vs. $2.3 \%(p=0.702$, $\mathrm{OR}=1.16,95 \%$ Cls: $0.55-2.46)$, respectively, showing a significant difference in admission rates, but not in mortality rates (Table 3 ). One patient was referred from the OPD for cellulitis with abscess formation and died of septic shock in the ED. She was admitted according to our established protocol.

Table 3

Admission and mortality rates of OPD-referral and self-referral.

\begin{tabular}{|llllll|}
\hline & $\begin{array}{l}\text { OPD-referral } \\
\mathrm{N}=564\end{array}$ & $\begin{array}{l}\text { Self-referral } \\
\mathrm{N}=564\end{array}$ & P-value & OR & 95\% Cls \\
\hline Hospitalization & & & & \\
\hline Admission to hospital & $281(49.8 \%)^{\#}$ & $163(28.9 \%)$ & $<0.001$ & 2.44 & $1.91 \sim 3.12$ \\
\hline Discharge from the ED & $283(50.2 \%)$ & $401(71.1 \%)$ & & \\
\hline Mortality & & & & \\
\hline Death & $15(2.7 \%)$ & $13(2.3 \%)$ & 0.702 & 1.16 & $0.55 \sim 2.46$ \\
\hline Survival to discharge & $549(97.3 \%)$ & $551(97.7 \%)$ & & \\
\hline OPD = outpatient department; OR = odds ratio; Cls = confidence intervals; ED = emergency department
\end{tabular}

We separated patients into emergency and urgent subgroups. For the emergency subgroup, the admission and mortality rates of the OPD-referral group and the self-referral group were $62.6 \%$ vs. $55.8 \%$ $(p=0.257, O R=1.33,95 \%$ Cls: $0.81-2.16)$ and $4.6 \%$ vs. $8 \%(p=0.253, O R=0.55,95 \%$ Cls: 0.20 to 1.54$)$, respectively (Table 4 ). 
Table 4

Admission and mortality rates of emergency patients.

\begin{tabular}{|llllll|}
\hline & $\begin{array}{l}\text { OPD-referral } \\
\text { N=131 }\end{array}$ & $\begin{array}{l}\text { Self-referral } \\
\text { N=138 }\end{array}$ & P-value & OR & 95\% Cls \\
\hline Hospitalization & & & & \\
\hline Admission to hospital & $82(62.6 \%)^{\#}$ & $77(55.8 \%)$ & 0.257 & 1.33 & $0.81 \sim 2.16$ \\
\hline Discharge from the ED & $49(37.4 \%)$ & $61(44.2 \%)$ & & & \\
\hline Mortality & & & & \\
\hline Death & $6(4.6 \%)$ & $11(8 \%)$ & 0.253 & $0.20 ~ 1.54$ \\
\hline Survival to discharge & $125(95.4 \%)$ & $127(92 \%)$ & & \\
\hline OPD = outpatient department; OR = odds ratio; Cls = confidence interval; ED = emergency department & \\
\hline \# One patient referred by the OPD died in the ED and was included as an admission & \\
\hline
\end{tabular}

For the urgent subgroup, the OPD-referral and the self-referral groups had a significant difference regarding the admission rate ( $46 \%$ vs. $20.2 \%, p<0.001$; OR $=3.36,95 \%$ Cls: 2.48 to 4.55 ) (Table 5). The OPD referrals trended to have a higher mortality rate but revealed no significant difference $(2.1 \%$ vs. $0.5 \%$, $p=0.064 ;$ OR $=4.50,95 \%$ Cls: $0.96-20.95)$.

Table 5

Admission and mortality rates of urgent patients.

\begin{tabular}{|llllll|}
\hline & $\begin{array}{l}\text { OPD-referral } \\
\text { N=433 }\end{array}$ & $\begin{array}{l}\text { Self-referral } \\
\mathbf{N}=426\end{array}$ & P-value & OR & 95\% Cls \\
\hline Hospitalization & & & & \\
\hline Admission to hospital & $199(46 \%)$ & $86(20.2 \%)$ & $<0.001$ & 3.36 & $2.48 \sim 4.55$ \\
\hline Discharge from the ED & $234(54 \%)$ & $340(79.8 \%)$ & & & \\
\hline Mortality & & & & & \\
\hline Death & $9(2.1 \%)$ & $2(0.5 \%)$ & 0.064 & 4.50 & $0.96 ~ 20.95$ \\
\hline Survival to discharge & $424(97.9 \%)$ & $424(99.5 \%)$ & & & \\
\hline OPD = outpatient department; OR = odds ratio; Cls = confidence interval; ED = emergency department \\
\hline
\end{tabular}

To compare the LoS in the ED, we further divided the OPD-referral and self-referral patients into subgroups according to whether they were considered emergency or urgent and whether they were or were not admitted (Table 6). For the admitted patients, all patients waited until the ward or ICU was available, regardless of whether they were emergency patients or urgent patients, and there was no 
significant difference between the OPD and self-referral groups. For the emergency-discharge subgroups, there was no significant difference between the OPD- and self-referral groups since emergency patients usually needed more examinations and took longer to regain a stable condition. In the urgent-discharge subgroup, there was significant differences between the OPD- and self-referral groups $(p<0.001)$, with median LoS of 5.8 hours versus 2.3 hours (Table 6).

Table 6

Comparison of length of stay between the OPD-referral and self-referral groups by subgroup.

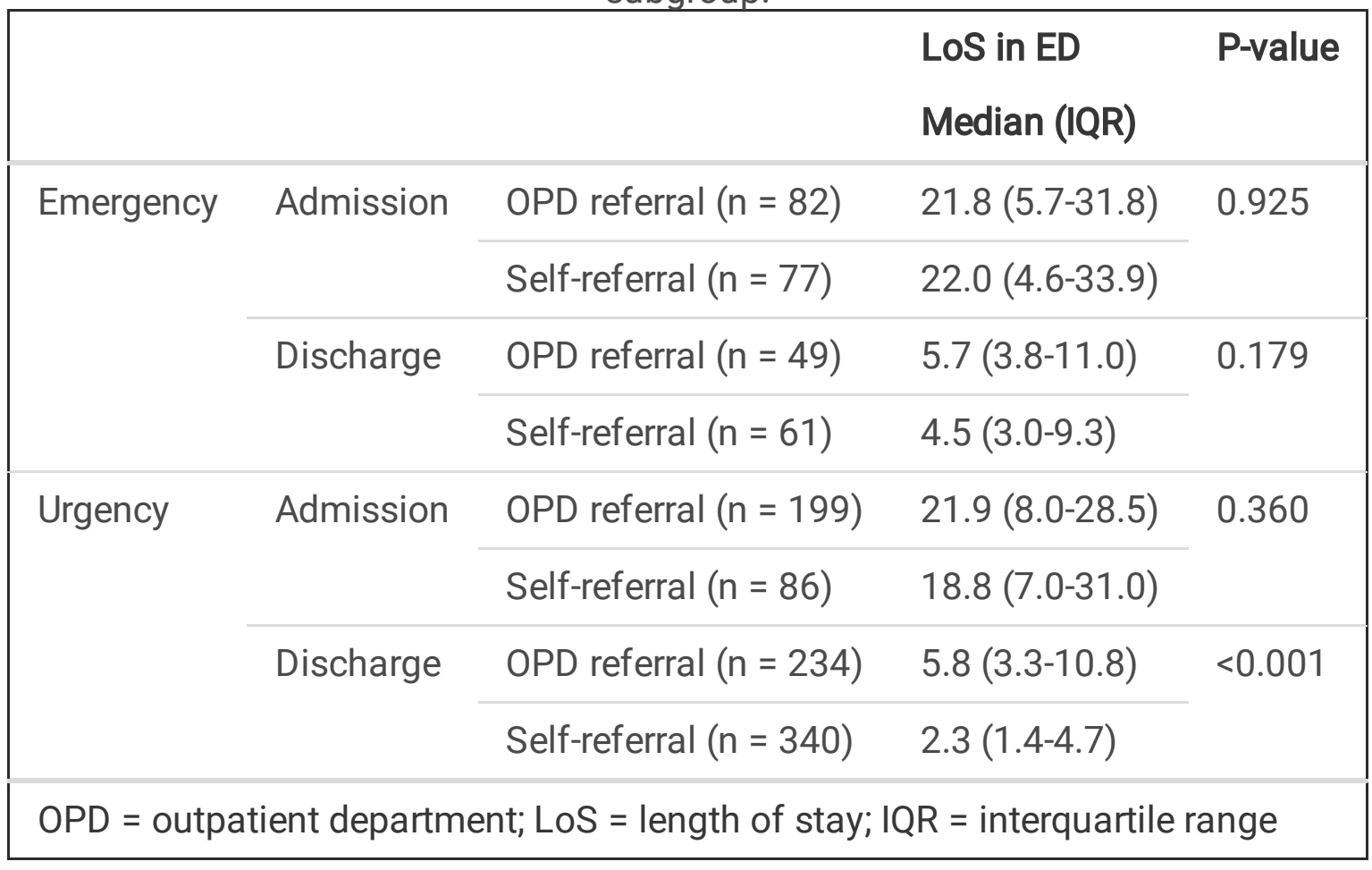

\section{Discussion}

EDs are designed to provide emergency care. They are not ideal locations for primary care because there is often no continuity of care, there is a risk of unnecessary testing, and an ED visit is more costly than a primary care visit [8]. Patients may often be unable to judge the severity of their condition and may view nonurgent symptoms as urgent, leading them to seek urgent medical care at the ED with the expectation of further investigation [8]. Some of these patients' issues are better suited to an LMD or OPD visit because they are relatively chronic and require a long adjustment period. However, there are some situations for which patients will be referred by an OPD; for example, when the patient has problems that cannot be resolved by the OPD because more observation time is necessary, and sometimes emergency or urgent examinations are needed to rule out acute illness and manage the illness. There is a difference between OPD-referred and self-referred patients: the OPD-referred patients have already been seen by a doctor who knows their condition well and has defined it as medically necessary. In most cases, patients were referred to the ED by the OPD for specific reasons, such as to receive a CT or MRI, undergo blood transfusion, prepare for surgery, or even be directly admitted to the hospital. 
Demographic analysis revealed that the OPD-referral group was older, had lower percentages of lessurgent and nonurgent patients, and had a higher percentage of patients with comorbidities than the selfreferral group. The reason for this difference is that most of the patients in the OPD-referral group were being regularly followed at the OPD for these underlying diseases, while most of the self-referral patients sought care at the ED for new problems. This result is consistent with previous studies that showed that the most common reasons for ED visits were extremity injuries, toothache, and medication refill requests, which did not require hospitalization [9].

Since these two groups had different patient compositions, we excluded inconsistent factors and matched self-referral patients with OPD referral patients with the same demographic profile, triage level, qSOFA score and major comorbidities. We then compared these two similar groups. The admission rates were $49.8 \%$ vs. $28.9 \%$ for OPD referrals and self-referrals, which was significantly different $(p<0.001)$ (Table 3). When emergency and urgent subgroups of patients were compared separately, we found that the difference in admission rates between OPD referrals and self-referrals arose mainly from urgent referrals $(46 \%$ vs. $20.2 \%, p<0.001)$ (Table 5$)$. A possible reason is that the patients in the OPD-referral group had more severe and complex medical conditions, and some of these patients were referred to the ED for admission because their conditions could not wait until they were able to visit the OPD.

The emergency subgroup of the self-referral group had a higher mortality rate than that of the OPDreferral group, $8 \%$ vs. $4.6 \%$. These 11 deaths included 7 patients with progression of malignancy. They were terminal malignancy cases receiving hospice care at home. They came to the ED for symptom and sign control and were usually admitted to the hospice ward. The remaining 4 patients had acute respiratory distress syndrome after a significant operation, massive stroke, type A aortic dissection and acute respiratory failure. In the urgent subgroup, the mortality rate was the opposite of that of the emergency subgroup, and the OPD-referral group had higher mortality rates $(2.1 \%$ vs. $0.5 \%, p=0.064)$. The OPD-referral group was referred by OPD doctors as medically urgent patients, and they had more severe and complex medical conditions. Therefore, the tendency of a higher mortality rate among OPDreferral patients should not be overlooked.

Regarding the LoS, there was no significant difference in admission between the emergency or urgent subgroups. The major factor in admission was whether there were available hospital beds, and the patient's referral and urgent status had little effect. Among the patients who were discharged, there was no significant difference in emergency subgroup, but in the urgent subgroup, OPD-referred patients had a longer length of stay in the ED before discharge. The reason for this discharge may be that for the OPDreferred patients, a doctor had determined their need to visit the ED, and they had visited the ED to address a specific situation, such as the control of disease symptoms and signs, and to undergo observation to determine the need for further examination or admission.

\section{Limitation}

There were some differences in characteristics between the OPD-referral and self-referral groups: Home hospice care patients came to the ED when their condition deteriorated, and they greatly affected the 
results because they comprised those who died in the ED, which may have caused an overestimation of the mortality rate for the self-referral group. Trauma cases account for a large percentage of ED visits, but we excluded them because there were a limited number of trauma-related OPD-referrals compared to selfreferrals and because most of the OPD-referred trauma patients had chronic wounds, whereas the selfreferred patients had fresh traumas. The inclusion of trauma cases in a more even manner could provide a better representation of ED visits in the future. Patients who were referred from LMD or other hospitals were excluded; although they had similar characteristics to the OPD-referral patients, they were excluded because some of them did not have laboratory test results or imaging copies, whereas patients referred by the OPD of our own hospital provided all information. Further inclusion of these patients could be considered if there was better transmission of information.

\section{Conclusion}

OPD-referral patients have a higher admission rate and longer length of stay than self-referral patients who are triaged as urgent. The urgent patients who were referred from OPD tend to have a higher mortality rate. We should be more alert to OPD-referred patients even when they initially appear not severely ill. Spending more time with these patients to identify dangerous problems is warranted.

\section{Abbreviations}

ED: emergency department

OPD: outpatient department

OR: odds ratio

Cl: confidence interval

qSOFA: Quick Sequential Organ Failure Assessment score

LoS: length of stay

IQR: interquartile range

TTAS: Taiwan Triage and Acuity Scale

OHCA: out-of-hospital cardiac arrest

\section{Declarations}

\section{-Ethic approval and consent to participate:}

The study was approved by the institutional review board of the National Cheng Kung University Hospital, Tainan, Taiwan (NCKUH-B-ER-110076). The informed consent was waived. 


\section{-Consent for publication:}

The consent of publication was not applicable.

\section{-Availability of data and material:}

The datasets used and analyzed during the current study are available from the corresponding author on reasonable request.

\section{-Competing interests:}

The authors declare that they have no competing interests.

\section{-Funding:}

The study was funded by Taiwan Ministry of Science and Technology (MOST 109-2634-F-006-023-).

\section{-Author's contributions:}

C.-H. Lin conceived the study. C.-H. Lin and C.-C. Hsieh developed the study protocols. Y.-R. Chou, M.-C. Ma, C.-C. Lee, and C.-C. Hsieh performed statistical analysis. Y.-R. Chou, C.-C. Hsieh and C.-H. Lin interpreted the study results. Y.-R. Chou, C.-C. Hsieh and C.-H. Lin drafted the manuscript. All authors contributed substantially to its revision. C.-C. Hsieh and C.-H. Lin are the corresponding authors who take responsibility for the paper as a whole. All authors read and approved the final manuscript.

\section{-Acknowledgements:}

Not applicable

\section{References}

1. Kraaijvanger N, Rijpsma D, van Leeuwen $H$, van Dijk N, Edwards M: Self-referrals in a Dutch Emergency Department: how appropriate are they? Eur J Emerg Med. 2016;23(3):194-202.

2. Sempere-Selva T, Peiró S, Sendra-Pina P, Martínez-Espín C, López-Aguilera I: Inappropriate use of an accident and emergency department: magnitude, associated factors, and reasons-an approach with explicit criteria. Ann Emerg Med. 2001;37(6):568-579.

3. Miyazawa A, Maeno T, Shaku F, Tsutsumi M, Kurihara H, Takayashiki A, et al.: Inappropriate use of the emergency department for nonurgent conditions: patient characteristics and associated factors at a Japanese hospital. J Gen Fam Med. 2019;20(4):146-153.

4. Pitts SR, Pines JM, Handrigan MT, Kellermann AL: National trends in emergency department occupancy, 2001 to 2008: effect of inpatient admissions versus emergency department practice intensity. Ann Emerg Med. 2012;60(6):679-686. e673. 
5. Selby JV, Fireman BH, Swain BE: Effect of a copayment on use of the emergency department in a health maintenance organization. N Engl J Med. 1996;334(10):635-642.

6. Catzikiris N, Tapley A, Morgan S, Van Driel M, Spike N, Holliday EG, Ball J, Henderson K, McArthur L, Magin P: Emergency department referral patterns of Australian general practitioner registrars: a cross-sectional analysis of prevalence, nature and associations. Aust health Rev. 2019;43(1):21-28.

7. Ringberg U, Fleten N, Førde OH: Examining the variation in GPs' referral practice: a cross-sectional study of GPs' reasons for referral. Br J Gen Pract. 2014;64(624):e426-e433.

8. Kraaijvanger N, van Leeuwen $\mathrm{H}$, Rijpsma D, Edwards M: Motives for self-referral to the emergency department: a systematic review of the literature. BMC Health Serv Res. 2016;16(1):685-685.

9. Diesburg-Stanwood A, Scott J, Oman K, Whitehill C: Nonemergent ED patients referred to community resources after medical screening examination: characteristics, medical condition after 72 hours, and use of follow-up services. J Emerg Nurs. 2004;30(4):312-317.

\section{Figures}

\section{Image not available with this version}

\section{Figure 1}

The patient flow diagram. \$ Data loss defined as patients without vital signs at triage which caused the incalculable of quick sequential organ failure assessment score (qSOFA). OPD: Outpatient department.

\section{Supplementary Files}

This is a list of supplementary files associated with this preprint. Click to download.

- TableS1.docx 